\title{
Ultrasound Assisted Liposuction for the Palliative Treatment of Madelung's Disease: A Case Report
}

\author{
Angela Faga, M.D., Luigi Antonio Valdatta, M.D., Alessandro Thione, M.D., and Mara Buoro, M.D.
}

Varese, Italy

\begin{abstract}
Benign Symmetric Lipomatosis (Madelung's disease) is a rare disease, characterized by massive fatty deposits in the neck, the shoulders, and the upper extremities. The deformity is associated with chronic alcohol use, malignant tumors of the upper airways, neuropathy, diabetes mellitus, hyperlipidemia, and other metabolic disorders. Although the deformity is prone to recurrence, surgical removal via lipectomy or liposuction provides the only way of palliation.

This paper describes the treatment of a 51-year-old man with a history of alcoholism and liver cirrhosis. He reported masses in his cervical and facial regions that had gradually enlarged over a period of 6 years. He also developed respiratory symptoms due to the fatty compression of his upper airways. Our experience with ultrasound-assisted liposuction for the palliative treatment of this disease is reported.
\end{abstract}

Key words: Madelung's disease-Ultrasound assisted liposuction

We present a case of Benign Symmetric Lipomatosis treated with ultrasound-assisted liposuction.

Benign symmetric lipomatosis (BSL), also known as Madelung's disease or Launois-Bensaude disease, is a rare condition of middle age, typical of males between 40 and 50 years old. It is characterized by multiple, symmetrical, unencapsulated fatty accumulations in the neck, shoulders, and upper extremities $[1,2,3]$. In the longstanding disease, the massive fatty deposits in the neck can cause compression of the aerodigestive tract and cava vein compression with mediastinic syndrome [1].

The deformity is associated with chronic alcohol use,

Correspondence to Faga Angela, M.D., Plastic Surgery Unit, Viale Borri 57, 21100 Varese-Italy, email: chirplas@libero.it malignant tumors of the upper airways [2, 3, 4], and also with peripheral neuropathy $[1,5]$. The aetiology of Madelung's disease is still discussed. It has been frequently reported in association with systemic metabolic abnormalities, like diabetes mellitus, hyperlipidemia, hyperuricemia, and a familiar character $[3,4]$.

The most recent hypotheses acknowledge Madelung's lipomatosis as an expression of an alcohol-induced endocrine disorder [6]; since lipid metabolism is closely related to mitochondrial respiration, biochemical studies [7] on muscle and adipose cells [5] have shown evidence of mitochondrial respiratory enzymes dysfunction. Electrophysiological measures displayed signs of central and peripheral nervous system involvement [5], while molecular genetic analysis revealed multiple deletions of mitochondrial DNA in muscle and adipose cells.

Surgical removal of fatty deposits is the only real hope of palliation. However, cases of volumetric regression of masses have also been described in literature [1] after the correction of nutritional deficiency and the stop of alcohol abuse.

\section{Case Report}

In the present paper the case of a 51-year-old man with a history of smoking (30 cigarettes per day) and alcoholism, with a negative anamnesis for cardiovascular and metabolic diseases, is described. Physical check-up, diagnostic, and laboratory examinations revealed liver cirrhosis, chronic erosive gastritis, diverticolosis sigmae, ernia jatale, gallstones, and chronic bronchitis.

The man reported fatty masses, which had enlarged gradually in his cervicofacial region over a period of 6 years (Fig. 1). He also reported fatty masses that involved the shoulders and, to a smaller degree, the upper extremities and the upper third of the thorax.

The patient developed aerodigestive symptoms which 


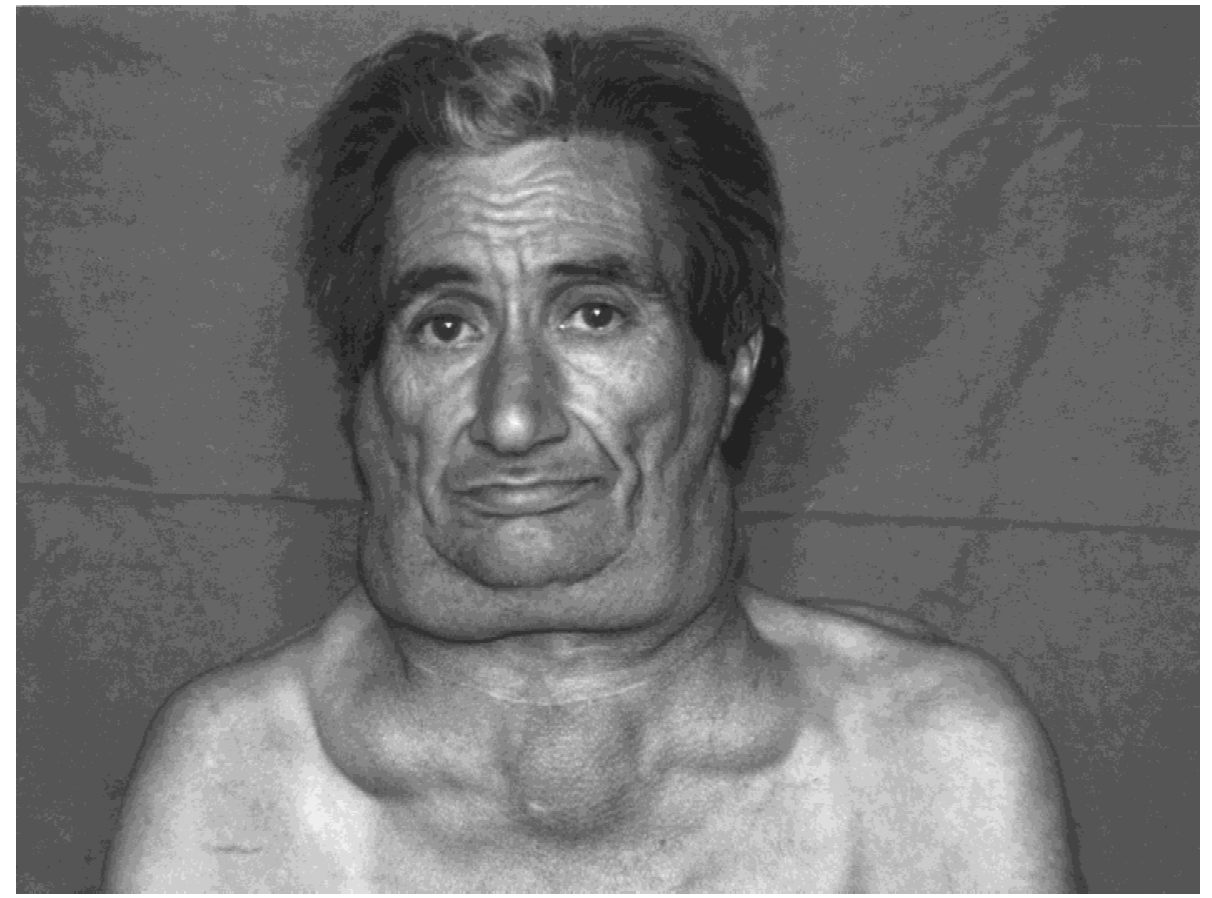

Fig. 1. Patient affected by Madelung's disease, preoperative view. were not only due to chronic bronchitis or gastrointestinal diseases, but mainly due to the fatty compression of the upper airway resulting in a stifling sense. A carcinoma of the upper airway was excluded with endoscopy and imaging.

The patient had undergone an anterolateral cervical lipectomy three years earlier, but the masses had relapsed after three months.

A preoperative echography of the neck and of the mandibular area confirmed the presence of multiple subfascial and prefascial fatty accumulations.

After having carried out preoperative medical clearance under general anesthesia, ultrasound assisted liposuction of laterocervical, paracervical, and perimandibular masses was planned.

All procedures were performed using Sculpture SMEI ${ }^{\circledR}$ Ultrasound Liposculpturing, which consists of: an electrical power console, a hand-piece that converts electrical energy to ultrasonic energy, and a blunt, ultrasound titanium probe $(15 \mathrm{~cm}$ long with a $0.3-\mathrm{cm}-$ long diameter). The titanium probe was connected to the hand-piece and delivered ultrasonic energy to the tissues. After this application a pump (Pompa IP 16 SMEI $^{\circledR}$ ) was used in order to draw up the emulsion produced. The patient had signed an informed assent before the operation.

The anatomical pattern of the areas to be treated was altered in a similar way by lipomatosis and by former surgery. In order to minimize traumatic effects on delicate deep structures of the neck caused by applying the ultrasound waves to the wrong anatomical sites, a right submandibular incision of $5 \mathrm{~cm}$ was performed. The incision was parallel to the mandibular rim and $1 \mathrm{~cm}$ beneath it. It revealed the presence of two distinct cervical pads: preplatysmal and subplatysmal. On these two pads the ultrasound probe was applied with an open approach. In so doing, the fat tissue was emulsified. Some of which was aspirated and some of which was removed with moist gauzes.

On the left side a conventional close-approach ultrasonic liposuction was performed by making $0.5-\mathrm{cm}-$ long preauricolar and submental incisions. A blunt cannula (15 cm long, with a 0.3 -cm-long diameter) was introduced for tunneling, and was moved respecting, to a great extent, the anatomic structures symmetrically arranged to the right side (Fig. 2).

Both right and left side were injected with epinephrine $1 \times 10^{6}$ in $0.9 \%$ saline solution; ultrasound liposuction was carried out and a total of $300 \mathrm{cc}$ was aspirated from the neck and the face.

Two suction drains were positioned, respectively, on the left and on the right and site-access incisions were closed with a single nylon suture. Subsequently, gauze dressing and elastic moderate-compression garments were applied. The surgery lasted two hours.

Perioperative intravenous single-dose prophylactic antibiotic (ceftriaxone, $2 \mathrm{~g}$ ) was prolonged for ten days with a half dosage $(1 \mathrm{~g})$ until drain removal. Each drain collected $150 \mathrm{cc}$ of fluid material which was submitted for a cytological test. The test suggested the presence of blood, injection fluid, and liquid adipose tissue.

The patient was discharged from the hospital 15 days after the operation. In the first two weeks after the surgery, the massive inflammatory response disguised the real result of the liposculpture and caused pain in the treated regions. However, this symptomatology spontaneously resolved in one month after drain removal.

Six months later this treatment provided only a modest 


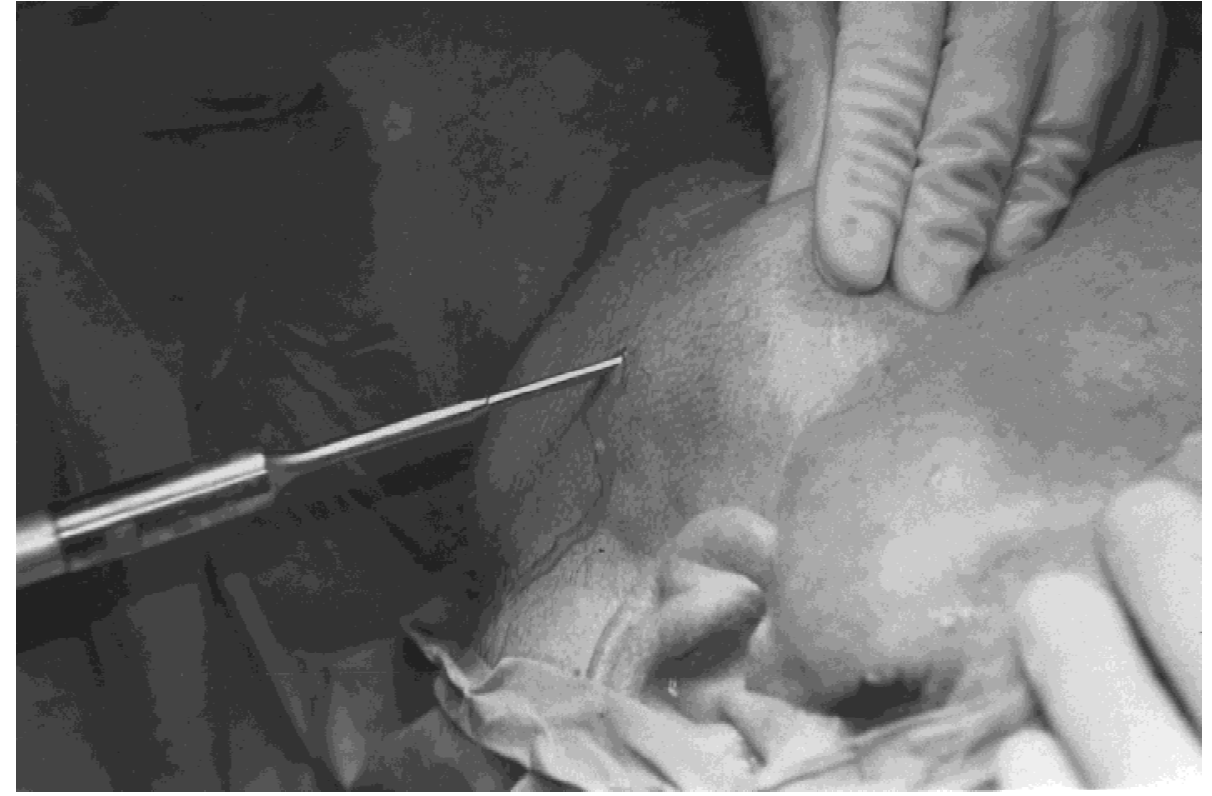

Fig. 2. I.O. view: ultrasound probe introduced into the left laterocervical region. Liquefied fat is flowing out. cosmetic result, but the aerodigestive symptoms improved significantly and the patient was satisfied.

Unfortunately, follow-up at 1 year showed a complete recurrence of both the symptoms and the disfigurement of body contour. This was obviously linked to the fact that the patient was still a smoker and an alcohol-abuser.

\section{Discussion and Conclusions}

As reported in current literature [8], the treatment of Madelung's disease aims at palliative results, at the relief of the sense of stifling and the inability to move the neck freely. The only effective way of healing is the surgical removal of fatty tissue, either by direct excision of the masses (resulting in long skin incisions, skin undermining, and relevant bleeding), or by a less aggressive liposuction.

According to the medical history and the clinical state of the man, an ultrasound liposculpture was performed. The patient was in a poor state of health, a smoker and a long-standing alcohol abuser; cirrhotic with a high anaesthesiologist risk (ASA 4), and scoagulated blood. In such cases, non-"quoad vitam" surgery can be carried out only if considered not dangerous, not too aggressive, and technically easy enough to be stopped at any moment. A radical laterocervical lipectomy, more aggressive and more technically difficult, might not have prevented other recurrences [8]. Compared with traditional liposuction, the ultrasound technique allows the surgeon to use more delicate and precise movements. It also allows much of the refined vascular and connettival framework of the subcutaneous tissue to be saved, reduces intraop- erative and postoperative bleeding, and shortens operative time.

This operation was carried out to relieve the simptomatology rather than to improve the body contour disfigurement, which was so severe that it limited interpersonal relationships. Six months after the operation the result was good, but one year later a complete recurrence took place, probably due to the patient's unwillingness to avoid smoking and alcohol.

\section{References}

1. Amicucci G, Sozio ML, Rizzo FM, Sozio A: Madelung's disease: Clinical case and review of the literature. Minerva Chir 53(7-8):655, 1998

2. Chan ES, Ahuja AT, Lau WY: Head and neck cancers associated with Madelung's disease. Ann Surg Oncol 6(4): 395, 1999

3. Enziger F, Weiss SW: Benign Lipomatous Tumors in Soft Tissue Tumors, Mosby: St. Louis, 1999

4. Klopstock T, Naumann M, Seibel P, et al: Mitochondrial DNA mutations in multiple symmetric lipomatosis. Mol Cell Biochem 174(1-2):271, 1997

5. Knopper D, Feidt H, Hornberger W: Madelung's lipomatosis of the neck-Expression of an alcohol-induced endocrine disorder? HNO 34(11):474, 1996

6. Smith PD, Stadelmann WK, et al: Benign symmetric lipomatosis (Madelung's disease). Ann Plast Surg 41(6):671, 1998

7. Tizian C, Berger A, Vykoupil KF: Malignant degeneration in Madelung's disease (benign lipomatosis of the neck): Case Report. Br J Plast Surg 36(2):187, 1983

8. Wu TP, Tsai JG, Chan PH, et al: Mitochondrial respiratory function in multiple symmetrical lipomatosis: Report of two cases. J Formos Med Ass 93(6):513, 1994 